\title{
Safer aortic crossclamping during short-term moderate hypothermic circulatory arrest for cardiac surgery in patients with a bad ascending aorta
}

\author{
Yoshiyuki Takami, MD, Kazuyoshi Tajima, MD, Sachie Terazawa, MD, Noritaka Okada, MD, Kei Fujii, MD, and \\ Yoshimasa Sakai, MD
}

\begin{abstract}
Objective: Cardiac surgery in patients with severely atherosclerotic or porcelain ascending aorta is technically challenging, with markedly increased risk of atheroembolism. We describe a technique of meticulous crossclamping of a difficult aorta during short-term moderate hypothermic circulatory arrest.
\end{abstract}

\begin{abstract}
Methods: From 1997 to 2007, we found 40 patients (mean age, $70 \pm 8$ years), including 14 patients undergoing hemodialysis, whose preoperative computed tomographic and intraoperative epiaortic ultrasonographic scans revealed eggshell calcification $(n=15)$ or protruding atheromas $(n=25)$ of the ascending aorta. They underwent cardiac surgery (aortic, 31 patients; mitral, 3 patients; both, 5 patients; and coronary alone, 1 patient) by means of meticulous crossclamping during hypothermic circulatory arrest for $3.4 \pm 1.5$ minutes at a rectal temperature of $29.0^{\circ} \mathrm{C} \pm 2.3^{\circ} \mathrm{C}$. During hypothermic circulatory arrest, we performed only internal inspection to identify the safe location of crossclamping in 21 patients, whereas we required debridement of calcification or atheroma by using the Cavitron Ultrasonic Surgical Aspirator (Tyco Healthcare, Mansfield, Mass) for safe crossclamping in 19 patients.
\end{abstract}

\begin{abstract}
Results: By using this technique, no patients died during the hospital stay. Stroke occurred in $1(2.5 \%)$ patient, and transient agitation occurred in 1 patient. Re-exploration for bleeding was required in 1 patient, and wound infection occurred in 2 patients. During follow-up, with a median time of 5.2 years, the overall survival rates were $100 \%, 90 \%$, and $76 \%$ at 1,3 , and 5 years, respectively. Three patients required reoperations during the follow-up period because of pseudoaneurysm in 2 patients and prosthetic valve infection in 1 patient.
\end{abstract}

Conclusion: Short-term moderate hypothermic circulatory arrest is quite useful for safe aortic crossclamping after internal inspection or debridement in high-risk patients with a severely atherosclerotic aorta.

Atherosclerotic disease of the ascending aorta is an important cause of stroke and embolic events after cardiac surgery. ${ }^{1-3}$ Aortic crossclamping is associated with a substantial release of particulate matter that has embolic potential. $^{4,5}$ This problem is increasingly encountered in the current practice of adult cardiac surgery, especially in elderly patients and those undergoing hemodialysis. Isolated coronary artery bypass grafting $(\mathrm{CABG})$ can be done without aortic manipulation. Mitral and tricuspid valve surgery can be performed without aortic crossclamping, sometimes with hypothermic fibrillation. However, patients who require aortic valve surgery cannot be managed with these techniques. Surgical strategies in such patients with a severely diseased ascending aorta include apicoaortic conduit, ${ }^{6}$ endoaortic balloon occlusion, ${ }^{7}$ aortic valve replacement (AVR) during profound hypothermic circula-

\footnotetext{
From the Department of Cardiovascular Surgery, Nagoya Daini Red Cross Hospital, Nagoya, Japan.

Received for publication May 28, 2008; revisions received Aug 28, 2008; accepted for publication Sept 10, 2008.

Address for reprints: Yoshiyuki Takami, MD, Department of Cardiovascular Surgery, Nagoya Daini Red Cross Hospital, 2-9 Myouken-cho, Showa-ku, Nagoya 4668650, Japan (E-mail: takami@nagoya2.jrc.or.jp).

J Thorac Cardiovasc Surg 2009; 137:875-80

$0022-5223 / \$ 36.00$

Copyright (c) 2009 by The American Association for Thoracic Surgery

doi:10.1016/j.jtcvs.2008.09.022
}

tory arrest (HCA), ${ }^{8}$ and local endarterectomy or total replacement of the ascending aorta during HCA to allow for the safe placement of a crossclamp followed by AVR. ${ }^{9,10}$ Unfortunately, the stroke rate, morbidity, and mortality are still high with these aggressive strategies. Considering that a less aggressive strategy is adequate in such high-risk patients with significant comorbidities, we have established a technique of meticulous aortic crossclamping during short-term moderate HCA followed by cardiac procedures. The purpose of this retrospective study is to report the results of our strategy for cardiac surgery in patients with a difficult ascending aorta.

\section{MATERIALS AND METHODS \\ Patients}

From January 1997 to December 2007, 40 patients were referred for cardiac surgery in the setting of atherosclerotic plaques $(n=25$, Figure 1$)$ or eggshell calcification ( $\mathrm{n}=15$, Figure 2 ) of the ascending aorta. They were $2.4 \%$ of 1645 adult patients who underwent cardiac surgery during the period in our institute. Their demographic data are listed in Table 1. Mean age was $70 \pm 8$ years (range, $44-83$ years), and 55\% were men. Seventy-six percent had pure aortic stenosis, and $14 \%$ had mixed aortic stenosis and regurgitation. Forty percent had coronary artery disease, and $20 \%$ had mitral valve disease. Fourteen $(35 \%)$ patients were receiving regular hemodialysis because of end-stage renal failure. One patient had a previous cardiac operation: CABG. All patients had preoperative evaluation of their entire thoracic aorta with computed tomographic scanning to examine the possible site of cannulation and crossclamping. 


\section{Abbreviations and Acronyms \\ $\mathrm{AVR}=$ aortic valve replacement \\ $\mathrm{CABG}=$ coronary artery bypass grafting \\ $\mathrm{CPB}=$ cardiopulmonary bypass \\ CUSA $=$ Cavitron Ultrasonic Surgical Aspirator \\ HCA = hypothermic circulatory arrest}

\section{Operative Procedures}

After standard anesthetic preparation, a median sternotomy was performed to expose the ascending aorta and the heart. The diagnosis of porcelain aorta, which had been detected on the preoperative computed tomographic scans, was confirmed by means of digital palpation of the aorta. The aorta was then examined by using epiaortic ultrasonographic scanning. Epiaortic scanning was performed with a $7.5-\mathrm{MHz}$ linear ultrasonic probe coated with ultrasonic gel packed in a sterile plastic sleeve. The scanning was first performed before opening the pericardium to sustain an echolucent window for optimal visualization of the anterior ascending aortic wall. The scanning was again performed after opening the pericardium to determine whether the protruding atheromatous plaque or mobile atheroma was present in the proposed region of cannulation and crossclamping. The patients with patchy calcification or atheroma plaques, in whom simply moving the crossclamp away from the involved sites sufficed, were excluded from this study cohort.

Aortic cannulation was the preferred site if a plaque-free and soft area was identified. Alternatively, the axillary or femoral arteries were used, and femoral cannulation was the last choice. The right atrium was cannulated directly for venous drainage, whereas bicaval cannulations were used in mitral valve surgery, and a common femoral venous cannulation of the right atrium was used in reoperations. Patients were then started on cardiopulmonary bypass (CPB) and cooled to a rectal temperature of approximately $30^{\circ} \mathrm{C}$ (an esophageal temperature of approximately $28^{\circ} \mathrm{C}$ ). A left ventricular vent was inserted through the right superior pulmonary vein. Once the appropriate temperature was reached, HCA was initiated with the patient in a steep Trendelenburg position. During HCA, the aortic cannula was clamped to avoid filling with air, the ascending aorta was opened in the line for AVR and not transected, and retrograde cold blood cardioplegia was administered. When the ascending aorta was inspected from inside and a suitable site for crossclamping was identified under direct vision, the aorta was crossclamped carefully, with blood flushed by means of retrograde cerebral perfusion by keeping the central venous pressure at approximately $20 \mathrm{~mm} \mathrm{Hg}$, followed by restart of perfusion through the aortic cannula. In patients with porcelain aorta, protruding plaques, or mobile atheroma, the subsequent crossclamp site was carefully cleared by means of debridement or endarterectomy with the Cavitron Ultrasonic Surgical Aspirator (CUSA; Tyco Healthcare, Mansfield, Mass). The debridement or endarterectomy was performed until the inner surface of the aorta to be clamped was judged to be smooth and soft. After washout by means of retrograde cerebral perfusion and disappearance of the floating debris or particles on the blood, circulation was restarted by slowly expelling air and debris from the ascending aorta, and the endarterectomized aorta was crossclamped. Once the aorta was crossclamped, full flow was resumed, rewarming was started, and the intracardiac procedures were performed. During this phase, myocardial protection was achieved by means of antegrade direct coronary ostial injection or retrograde coronary sinus delivery. Finally, the aorta was closed with double $4-0$ polypropylene sutures, with or without Teflon felt strips.

\section{Data Analysis}

This study was approved by the institutional review board of our hospital. Clinical, procedural, and postoperative data were collected for this retrospective review. Deaths occurring at any time during the hospital stay or

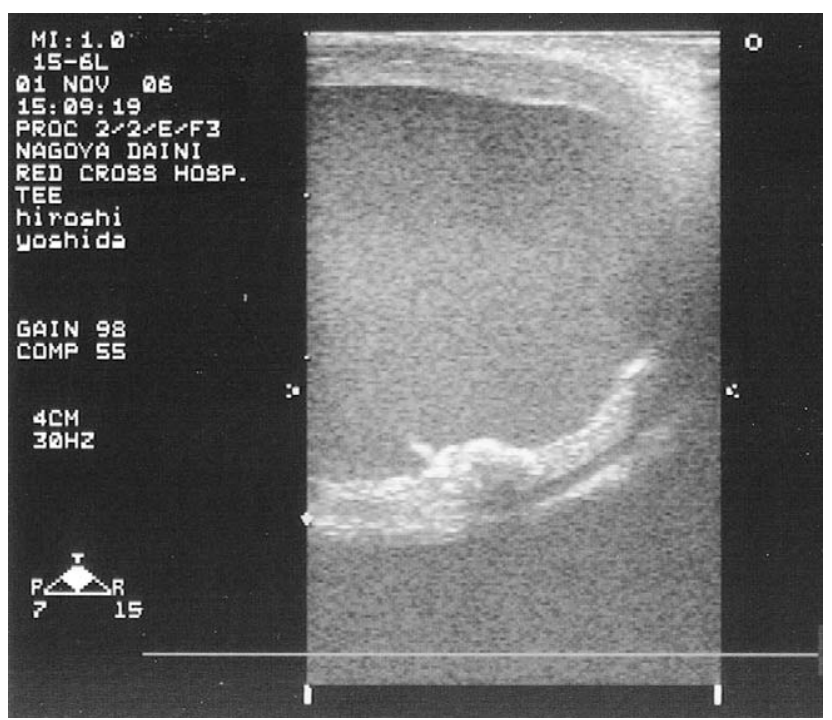

FIGURE 1. An intraoperative epiaortic ultrasonographic scan in a 70-yearold female patient who underwent aortic valve replacement with a $19-\mathrm{mm}$ Carpentier-Edwards Pericardial valve. Thick aortic intima over $2 \mathrm{~mm}$ and protruding atheroma are shown, suggesting the aorta is not unclampable.

anywhere within 30 days since surgical intervention were categorized as early deaths. Stroke was defined as a prolonged ( $>72$ hours) or permanent neurologic deficit not present preoperatively and persisting at the time of discharge. It is usually associated with abnormal results of magnetic resonance imaging or computed tomographic scans. Nonfocal neurologic derangements, such as confusion and psychosis, were recorded as neurocognitive defects. Neurologic assessment was performed by consulting neurologists, if necessary. Follow-up telephone interviews were conducted by the authors early in April 2008. Descriptive statistics were expressed as the mean \pm standard deviation. Kaplan-Meier survival curves were developed with StatView 5.0 software (Abacus Concepts, Inc, Berkeley, Calif).

\section{RESULTS}

A majority of 40 study patients underwent AVR $(n=36)$, as demonstrated in Table 2 . Among the patients who underwent AVR, 38\% had mechanical valves, and $62 \%$ had bioprostheses. The mechanical valves implanted included the CarboMedics (CarboMedics, Inc, Austin, Tex) 21-mm $(\mathrm{n}=2)$ and $23-\mathrm{mm}(\mathrm{n}=1)$ valves; the ATSAP (ATS Medical, Inc, Minneapolis, Minn) $18-\mathrm{mm}(\mathrm{n}=4)$ and 22-mm $(\mathrm{n}=1)$ valves; the Bicarbon 21-mm valve $(\mathrm{n}=3$; Sorin Biomedica Cardio, Saluggia, Italy); the OnX (Medical Carbon Research Institute, LLC, Austin, Tex) 19-mm $(\mathrm{n}=1)$ and 21-mm $(\mathrm{n}=2)$ valves; and the St Jude Medical regent 17$\mathrm{mm}$ valve ( $\mathrm{n}=1$; St Jude Medical, Inc, Minneapolis, Minn). The tissue valves implanted were the Hancock (Medtronic, Inc, Minneapolis, Minn) 21-mm $(\mathrm{n}=1), 23-\mathrm{mm}$ $(\mathrm{n}=1)$, and 25-mm $(\mathrm{n}=1)$ valves; the Mosaic (Medtronic, Inc) $23-\mathrm{mm}(\mathrm{n}=4)$ and $25-\mathrm{mm}(\mathrm{n}=1)$ valves; and the Carpentier-Edwards Pericardial (CEP; Baxter Healthcare Corp, Newport Beach, Calif) 19-mm $(\mathrm{n}=11), 21-\mathrm{mm}(\mathrm{n}=4)$, and $23-\mathrm{mm}(\mathrm{n}=2)$ valves. Aortic prostheses of less than $23 \mathrm{~mm}$ were implanted in $75 \%$ of the patients undergoing 


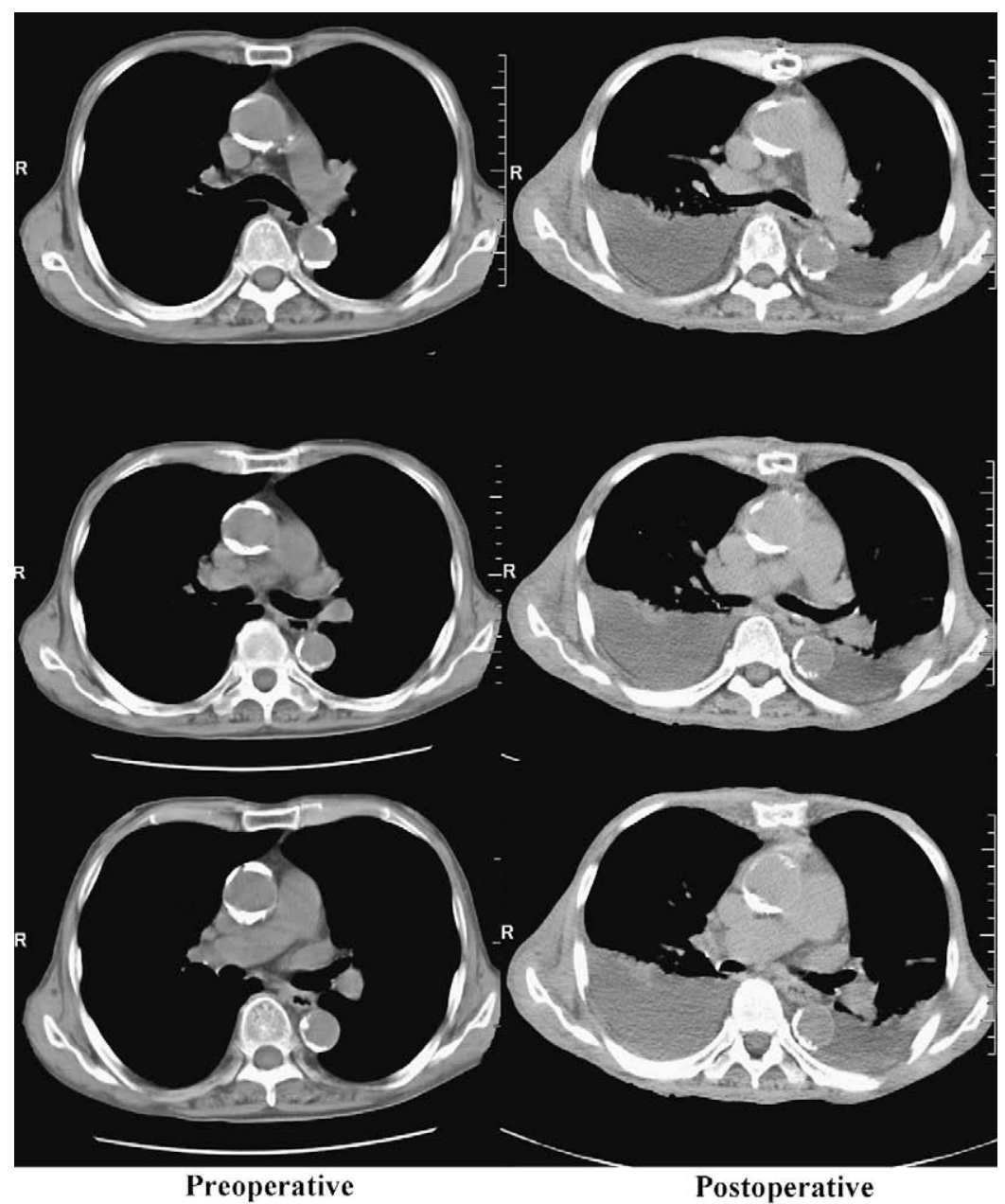

FIGURE 2. Computed tomographic scans before and after the operation in a 76-year-old man undergoing chronic hemodialysis for 18 years who underwent aortic valve replacement with annular dilatation with a 21-mm Carpentier-Edwards Pericardial valve. The diffuse calcification is specific to the patients undergoing hemodialysis, sometimes referred to a Monckeberg's sclerosis. The calcified plaques inside the aorta were removed with the Cavitron Ultrasonic Surgical Aspirator to make the aorta safe for crossclamping. The image shows that the volume of calcifications is decreased by the surgical maneuver.

AVR. Fifteen percent of the patients undergoing AVR required annular enlargement with a commercially available bovine or equine pericardium to obtain an effective orifice area of greater than $0.85 \mathrm{~cm}^{2} / \mathrm{m}^{2}$ and to avoid patient-prosthesis mismatch. Forty-two percent of patients undergoing AVR required additional CABG, with in situ arterial grafts only in 6 patients, saphenous vein grafts only in 3 patients, and both grafts in 6 patients. For CPB, as shown in Table 3 , the ascending aorta was cannulated in $70 \%$ of patients, whereas 30\% required alternative sites (axillary artery, $10 \%$; femoral artery, 20\%). The patients reached a rectal temperature of $29.0^{\circ} \mathrm{C} \pm 2.3^{\circ} \mathrm{C}$ and an esophageal temperature of $26.5^{\circ} \mathrm{C} \pm 2.6^{\circ} \mathrm{C}$ by means of systemic cooling for 15 \pm 8 minutes. No patients required a period of HCA for longer than 7.5 minutes, with a mean of $3.4 \pm 1.5$ minutes. During HCA, only inspection of the aorta from the inside was performed to identify a suitable site for crossclamping in $21(53 \%)$ patients, whereas additional debridement of calci- fication, protruding plaque, or mobile atheroma with CUSA was required in $19(47 \%)$ patients. The CPB duration and aortic crossclamp time averaged $228 \pm 67$ and $131 \pm 51$ minutes, respectively.

No patients died during the hospital stay. Postoperative hospital stay averaged $31 \pm 38$ days. The hospital stays of 3 patients undergoing hemodialysis were greater than 50 days (71 days, 163 days, and 199 days). Two of them had postoperative sternomediastinitis, which was fortunately cured completely. One patient had recurrent pleural effusion, resulting in longer hospital stay. When these patients were excluded, the mean hospital stay was $20 \pm 8$ days. Postoperative complications are highlighted in Table 4. There was 1 patient with a central neurologic complication, whose dysarthria and mild right hemiparesis were evident 2 days after AVR with a Mosaic 25-mm valve. In this patient the ascending aorta was crossclamped after inspection during $\mathrm{HCA}$ for 6 minutes at an esophageal temperature of 
TABLE 1. Demographics for 40 study patients

\begin{tabular}{lc}
\hline & No. $(\%)$ or mean \pm SD (range) \\
\hline Mean age (y) & $70 \pm 8(44-83)$ \\
Male/female sex & $22 / 18$ \\
Body surface area $\left(\mathrm{m}^{2}\right)$ & $1.58 \pm 0.21(1.26-1.96)$ \\
Hypertension & $27(60 \%)$ \\
Hyperlipidemia & $14(35 \%)$ \\
Smoking & $12(30 \%)$ \\
Diabetes mellitus & $9(23 \%)$ \\
Previous myocardial infarction & $10(25 \%)$ \\
Previous cerebrovascular accident & $2(5 \%)$ \\
Previous cardiac surgery & $1(2.5 \%)$ \\
Hemodialysis & $14(35 \%)$ \\
Duration (y) & $13 \pm 7(3-23)$ \\
Left ventricular ejection fraction $(\%)$ & $60 \pm 12(42-78)$ \\
NYHA class & \\
I & 0 \\
II & $17(43 \%)$ \\
III & $17(43 \%)$ \\
IV & $6(14 \%)$ \\
\hline
\end{tabular}

$S D$, Standard deviation; $N Y H A$, New York Heart Association.

$26^{\circ} \mathrm{C}$. Transient agitation and psychosis of milder degree was noted in only 1 patient. Re-exploration for bleeding was performed in 1 patient after reoperative AVR with a CEP 19-mm valve, in whom the sutures of annular enlargement with an equine pericardial patch was a bleeding site. Although $40 \%$ of patients required CABG, no patients had a documented perioperative myocardial infarction or low cardiac output necessitating circulatory assist.

Follow-up was obtained in all patients at a median interval of 5.2 years after surgical intervention $(3.6 \pm 2.4$ years; range, 57 days-10.4 years). Six late deaths occurred at 1.1, $1.7,2.0,3.2,5.0$, and 4.4 years because of cerebral infarction, gastrointestinal perforation, heart failure, bladder cancer, hepatoma, and pneumonia, respectively, leading to

TABLE 2. Surgical procedures

\begin{tabular}{lc}
\hline & No. $(\%)$ \\
\hline Procedure & \\
AVR alone & $18(45 \%)$ \\
AVR + CABG & $13(33 \%)$ \\
AVR + MVR & $1(3 \%)$ \\
AVR + MVP & $2(5 \%)$ \\
AVR + MVR + CABG & $2(5 \%)$ \\
AVR with annular enlargement & $6 / 36(17 \%)$ \\
Aortic prosthesis & \\
Mechanical/bioprosthetic & $15 / 21(38 \% / 62 \%)$ \\
Size $<23$ mm & $30(75 \%)$ \\
CEP 19 mm & $11(28 \%)$ \\
CABG & $1(3 \%)$ \\
MVR & $1(3 \%)$ \\
MVR + CABG & $1(3 \%)$ \\
MVP & $1(3 \%)$ \\
Urgent or emergency & $2(5 \%)$ \\
\hline AVR, Aotic vave
\end{tabular}

$A V R$, Aortic valve replacement; $C A B G$, coronary artery bypass grafting; $M V R$, mitral valve replacement; $M V P$, mitral valve plasty; $C E P$, Carpentier-Edwards Pericardial.
TABLE 3. Intraoperative data

No. $(\%)$ or mean \pm SD (range)

Arterial cannulation

Ascending aorta

Axillary artery

$28(70 \%)$

Femoral artery

$4(10 \%)$

$8(20 \%)$

Moderate hypothermia

Lowest esophageal temperature $\left({ }^{\circ} \mathrm{C}\right)$

Lowest rectal temperature $\left({ }^{\circ} \mathrm{C}\right)$

Circulatory arrest (min)

Manipulation of aorta

Inspection only

Debridement with CUSA

Cardiopulmonary bypass (min)

Aortic crossclamp time (min)

$26.6 \pm 2.6(22-31)$

$29.0 \pm 2.3(25-33)$

$3.4 \pm 1.5(1.0-7.5)$

$S D$, Standard deviation; CUSA, Cavitron Ultrasonic Surgical Aspirator

overall survivals of $100 \%, 90 \%$, and $76 \%$ at 1,3 , and 5 years, respectively (Figure 3 ). Three patients required reoperations during the follow-up period because of pseudoaneurysm in 2 patients and prosthetic valve infection in 1 patient. The pseudoaneurysm was observed at the suture line of the aortic patch, and the reoperations (ie, replacement of the ascending aorta) were performed 8 months and 44 months after the operation, respectively. The prosthetic valve infection was observed in a 66-year-old patient 5 months AVR and mitral valve replacement. All patients undergoing reoperations survived and remain alive.

\section{DISCUSSION}

Cerebral complications result in not only increased perioperative morbidity and mortality but also poor long-term prognosis after surgical intervention. ${ }^{3}$ Although the pathophysiology of stroke is multifactorial, we have to pay attention to cerebral microemboli during the operation. Several studies identified an association between the degree of atheroma in the ascending aorta and new brain ischemic

TABLE 4. Postoperative morbidity and mortality

\begin{tabular}{lc}
\hline & No. $(\%)$ or mean \pm SD (range) \\
\hline In-hospital death & 0 \\
Hospital stay (d) & $31 \pm 38(9-199)$ \\
Stroke & $1(2.5 \%)$ \\
Nonfocal neurologic derangement & $1(2.5 \%)$ \\
Re-exploration for bleeding & $1(2.5 \%)$ \\
IABP/low cardiac output & 0 \\
Perioperative myocardial infarction & 0 \\
Intubation $>48$ h & $4(10 \%)$ \\
ICU stay $>2$ d & $5(12.5 \%)$ \\
Wound infection & $2(5 \%)$ \\
Bowel disease & $2(5 \%)$ \\
Permanent pacemaker & 0 \\
Delayed reoperation & \\
Aortic pseudoaneurysm & $2(5 \%)$ \\
Prosthetic valve endocarditis & $1(2.5 \%)$ \\
\hline
\end{tabular}

$\overline{S D \text {, Standard deviation; } I A B P \text {, intra-aortic balloon pumping; } I C U \text {, intensive care unit. }}$ 


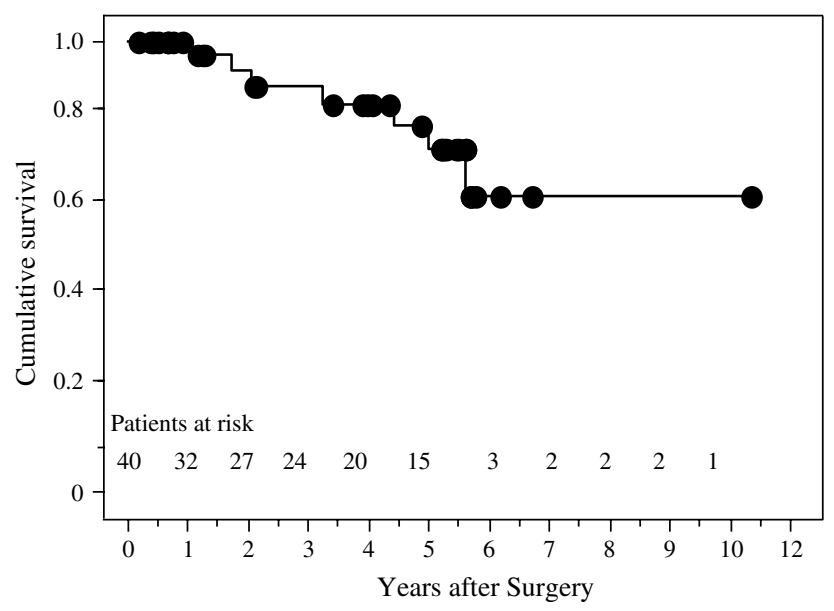

FIGURE 3. Kaplan-Meier estimate of overall survival.

lesions. ${ }^{2,11,12}$ As McKahann and associates ${ }^{12}$ addressed in a review article, the stroke incidence appears to be increasing because more high-risk patients currently undergo cardiac surgery, such as patients undergoing hemodialysis, whose vascular atherosclerosis is highly prevalent. ${ }^{13-15}$ In our patients $35 \%$ were undergoing hemodialysis. Calcification around atherosclerotic lesions increases plaque fragility, which in turn makes that part of the vessel wall more vulnerable to shear stresses, precipitating embolic complications after cardiac surgery, as focused on in the present study. Two distinct pathologic patterns of calcification are recognized. ${ }^{15}$ The first form is intimal calcification in the context of atherosclerotic plaque formation with lipid-laden form cells. This process is focal, and adjacent regions of the vessel wall might be normal. The second form of calcification is diffuse mineral deposition throughout the vascular tree, sometimes referred to a Monckeberg's sclerosis, as shown in Figure 2. This form of calcification occurs predominantly in the media of the vessel, with no relationship to atheromatous plaque formation. This is a striking feature of vascular disease seen in patients undergoing hemodialysis.

There have been several reports of surgical strategy to decrease manipulation of the unclampable aorta in cardiac surgery, as summarized by Zingone and colleagues. ${ }^{16} \mathrm{An}$ approach using an endoluminal occluding balloon was demonstrated to be ineffective and associated with a significantly higher risk of in-hospital death and stroke. ${ }^{7}$ Deep HCA $\left(<20^{\circ} \mathrm{C}\right)$ emerges as a core method to approach the difficult aorta during aortic valve surgery strictly requiring an aortotomy. The simplest approach appears to be AVR during deep HCA without aortic crossclamping. However, this technique was associated with high mortality and stroke rates of greater than $10 \% .{ }^{9,10}$ An alternative approach is aortic endarterectomy during deep HCA proceeding to aortic crossclamping. ${ }^{9,10}$ A more radical option is graft replacement of the atherosclerotic aorta during deep HCA. The results have been varied with a relatively lower stroke rates but a relatively high mortality rate. ${ }^{8-10}$

We are reluctant to use deep HCA as seen in previous reports because the patients with difficult aortas are at quite high risk and carry significant comorbidities. In particular, the patients undergoing hemodialysis experience malnutrition (low serum albumin), inflammation, and atherosclerosis syndrome, which are associated with all-cause and cardiovascular mortality. ${ }^{17}$ Therefore we have adopted moderate HCA $\left(28^{\circ} \mathrm{C}\right.$ to $\left.30^{\circ} \mathrm{C}\right)$ to simply perform aortic inspection or endarterectomy for safe aortic crossclamping in such high-risk patients. The maneuvers were intended to be as short and simple as possible. Compared with previous reports, the lowest temperature was much higher and the duration of HCA was much shorter in our study. We simply applied retrograde cerebral perfusion for brain protection during HCA, minimizing the risk of debris and air embolism. Although the approach has been simplified, both mortality and stroke rate in our study were superior to those seen in previous reports. In addition, we think that epiaortic ultrasonographic scanning is insufficient to guide safer clamp replacement and to determine whether the lesion should be endarterectomized. Direct inspection and aortic crossclamping under direct vision are more reliable and safer.

During moderate HCA, we proceeded with aortic endarterectomy or debridement, when needed, before safe aortic crossclamping. The atheromas with mobile components or calcified plaque were resected with the CUSA to make the aorta safe for clamping. One of the contributing factors to the lower mortality and stroke rate seen in our study might be the use of the CUSA. The CUSA is one of the most popular devices that facilitate bloodless liver transection. ${ }^{18} \mathrm{It}$ uses a vibrating metal tip to fragment tissue and then aspirates the debris through the hollow center of the tip. ${ }^{19}$ It has been also used in cardiac surgery, especially to remove valve annular calcification. ${ }^{20}$ Figure 2 demonstrates the comparison of the computed tomographic scans before and after the operation in a 76-year-old man undergoing chronic hemodialysis for 18 years who underwent AVR with annular dilatation with a 21-mm CEP valve. The calcified plaques inside the aorta were removed by using the CUSA to make the aorta safe for crossclamping. Figure 2 revealed that the volume of calcifications was decreased by using the surgical maneuver. Although our technique is effective for safer aortic crossclamping, we should pay attention to the fate of the endarterectomized aorta. In our patient cohort, 2 patients required reoperations during the follow-up period because of pseudoaneurysm formation. In both patients the pseudoaneurysm was observed at the suture line of the patch used for aortic annular enlargement and was also associated with infection. We should recognize the fragility of the aortic wall endarterectomized with the CUSA as a risk factor for pseudoaneurysm formation, and therefore we have to reinforce the aortic suture line carefully during the operation. 


\section{CONCLUSION}

Although conclusions from any nonrandomized, retrospective analysis of a small cohort of patients should be interpreted with caution, our technique of meticulous aortic crossclamping after internal inspection, endarterectomy, or both with the CUSA during short-term moderate HCA is useful to minimize the mortality and stroke rate in highrisk patients with a difficult ascending aorta.

\section{References}

1. van der Linden J, Hadjinikolaou L, Bergman P, Lindblom D. Postoperative stroke in cardiac surgery is related to the location and extent of atherosclerotic disease in the ascending aorta. J Am Coll Cardiol. 2001;38:131-5.

2. Mackensen GB, Ti LK, Phillips-Bute BG, Mathew JP, Newman MF, Grocott HP, et al. Cerebral embolization during cardiac surgery: impact of aortic atheroma burden. Br J Anaesth. 2003;91:656-61.

3. Filsoufi F, Rahmanian PB, Castillo JG, Bronster D, Adams DH. Incidence, topography, predictors and long-term survival after stroke in patients undergoing coronary artery bypass grafting. Ann Thorac Surg. 2008;85: 862-70.

4. Barbut D, Hinton RB, Szatrowski TP, Hartman GS, Bruefach M, WilliamsRusso $\mathrm{P}$, et al. Cerebral emboli detected during bypass surgery are associated with clamp removal. Stroke. 1994;25:2398-402.

5. Boivie P, Hansson M, Engström KG. Embolic material generated by multiple aortic crossclamping: a perfusion model with human cadaveric aorta. J Thorac Cardiovasc Surg. 2003;125:1451-60.

6. Lockowandt U. Apicoaortic valved conduit: potential for progress? J Thorac Cardiovasc Surg. 2006;132:796-801.

7. Zingone B, Gatti G, Rauber E, Pappalardo A, Benussi B, Dreas L. Surgical management of the atherosclerotic ascending aorta: is endoaortic balloon occlusion safe? Ann Thorac Surg. 2006;82:1709-14.

8. Byrne JG, Aranki SF, Cohn LH. Aortic valve operations under deep hypothermic circulatory arrest for the porcelain aorta: "no-touch" technique. Ann Thorac Surg. 1998;65:1313-5.
9. Gillinov AM, Lytle BW, Hoang V, Cosgrove DM, Banbury MK, McCarthy PM, et al. The atherosclerotic aorta at aortic valve replacement: surgical strategies and results. J Thorac Cardiovasc Surg. 2000;120:957-63.

10. Aranki SF, Nathan M, Shekar P, Couper G, Rizzo R, Cohn LH. Hypothermic circulatory arrest enables aortic valve replacement in patients with unclampable aorta. Ann Thorac Surg. 2005;80:1679-86.

11. Hangler HB, Nagele G, Danzmayr M, Mueller L, Ruttmann E, Laufer G, et al. Modification of surgical technique for ascending aortic atherosclerosis: impact on stroke reduction in coronary artery bypass grafting. $J$ Thorac Cardiovasc Surg. 2003;126:391-400.

12. McKhann GM, Grega MA, Borowicz LM Jr, Baumgartner WA, Selnes OA. Stroke and encephalopathy after cardiac surgery: an update. Stroke. 2006;37: 562-71.

13. Davies MR, Hruska KA. Pathophysiological mechanism of vascular calcification in end-stage renal disease. Kidney Int. 2001;60:472-9.

14. Qunibi WY, Nolan CA, Ayus JC. Cardiovascular calcification in patients with endstage renal disease: a century-old phenomenon. Kidney Int. 2002;82(suppl):S73-80.

15. Okuno S, Ishimura E, Kitatani K, Fujino Y, Kohno K, Maeno Y, et al. Presence of abdominal aortic calcification is significantly associated with all-cause and cardiovascular mortality in maintenance hemodialysis patients. Am J Kidney Dis. 2007; 49:417-25.

16. Zingone B, Rauber E, Gatti G, Pappalardo A, Benussi B, Forti G, et al. Diagnosis and management of severe atherosclerosis of the ascending aorta and aortic arch during cardiac surgery: focus on aortic replacement. Eur J Cardiothorac Surg. 2007;31:990-7.

17. Bechtel JF, Detter C, Fischlein T, Krabatsch T, Osswald BR, Riess FC, et al. Cardiac surgery in patients on dialysis: decreased 30-day mortality, unchanged overall survival. Ann Thorac Surg. 2008;85:147-53.

18. Lesurtel M, Selzner M, Petrowsky H, McCormack L, Clavien PA. How should transection of the liver be performed?: a prospective randomized study in 100 consecutive patients: comparing four different transection strategies. Ann Surg. 2005; 242:814-23.

19. Bond LJ, Cimino WW. Physics of ultrasonic surgery using tissue fragmentation. Ultrasonics. 1996;34:579-85.

20. Kellner HJ, Pracki P, Hildebrandt A, Binner C, Eisele G, Struck E. Aortic valve debridement by ultrasonic surgical aspirator in degenerative, aortic valve stenosis: follow-up with Doppler echocardiography. Eur J Cardiothorac Surg. 1996;10: 498-504. 